\title{
THE LAYERED STRUCTURE OF BONES OF BIRDS AS A POSSIBLE INDICATION OF AGE
}

\author{
by \\ R. W. M. VAN SOEST \& W. L. VAN UTRECHT \\ Zoological Laboratory, University of Amsterdam, The Netherlands
}

\begin{abstract}
A method of age determination, new in birds, is described. The method has been derived from similar methods commonly used to determine age of fishes, amphibians, reptilians, and mammals. It consists of demonstrating, by preparation of transverse transparent sections, the presence of annual deposits in the periosteal zone (= zone of outer circumferential lamellae) of bone. Evidence is presented that the layered structures in the periosteal zone are indeed annual and thus related with age.
\end{abstract}

\section{INTRODUCTION}

Periodical layering in hard structures of animals has been used for long to determine age of fishes. Otoliths, vertebrae, and scales grow continuously, but display periods of fast growth alternated with periods of slow growth, often appearing as wide and narrow, or light and dark bands. As yet, it is not fully clear which are the factors determining this periodical acceleration and deceleration of the growth of these structures. Possibly unfavourable conditions, for instance in winter, are responsible for this phenomenon; it is not unlikely that breeding also may influence growth.

Most of the important factors influencing growth are annual or seasonal (cold season - warm season, breeding season - non breeding season, etc.); this makes it possible to relate growth layers to age. This method has not only been applied in fishes, but also - though to a much smaller extent - in amphibians (Peabody, 1961) and reptilians (Petter-Rousseaux, 1953).

In homoiothermic vertebrates appliance of this method is somewhat more complicated. The main increase in length and weight is limited to the first (juvenile) stage of life, which takes a few weeks up to a few years according to the species, in contrast to poikilothermic animals which grow almost continuously throughout their whole life.
In the last decennia a very accurate method was developed for age determination of mammals, based on counting annual deposits, comparable to those of fishes, in the cementum and dentine of teeth. This method was first applied by Laws (1952) on Pinnipedia and his example was followed by many authors and with species of all mammalian orders. Later Russian biologists discovered that similar layering could also be found in the periosteal zone of mammalian bone. A complete review of all papers published on this subject, before 1967, and of all methods used to make the annual layers visible has been given by Klevezal \& Kleinenberg (1969).

Of the five vertebrate classes only birds have not been investigated upon the subject of annual layering in hard structures. In this short study the existence of layered structures in birds is demonstrated, and where possible, related to age.

\section{MATERIAL AND METHODS}

The material used for this study consisted of two specimens of Jay (Garrulus glandarius (L.)), one of Moorhen (Gallinula chloropus (L.)), five of Herring Gull (Larus argentatus Pontoppidan), one of Duck (Anas platyrhynchos L.), two of Chicken (Gallus gallus (L.)), and six of Heron (Ardea cinerea L.). Whenever possible the material is selected on the basis of commonly used age indications, mainly plumage characteristics. The Heron material thus consists of two "grey fronted" specimens (supposedly in the first year of their life), one "grey-white fronted" specimen (supposedly in its second year) and three "white fronted" specimens (supposedly over two years old). Of the five Herring Gulls, three have the first year plumage, one has the second year plumage and one the third year plumage. The two Chickens are guaranteed 


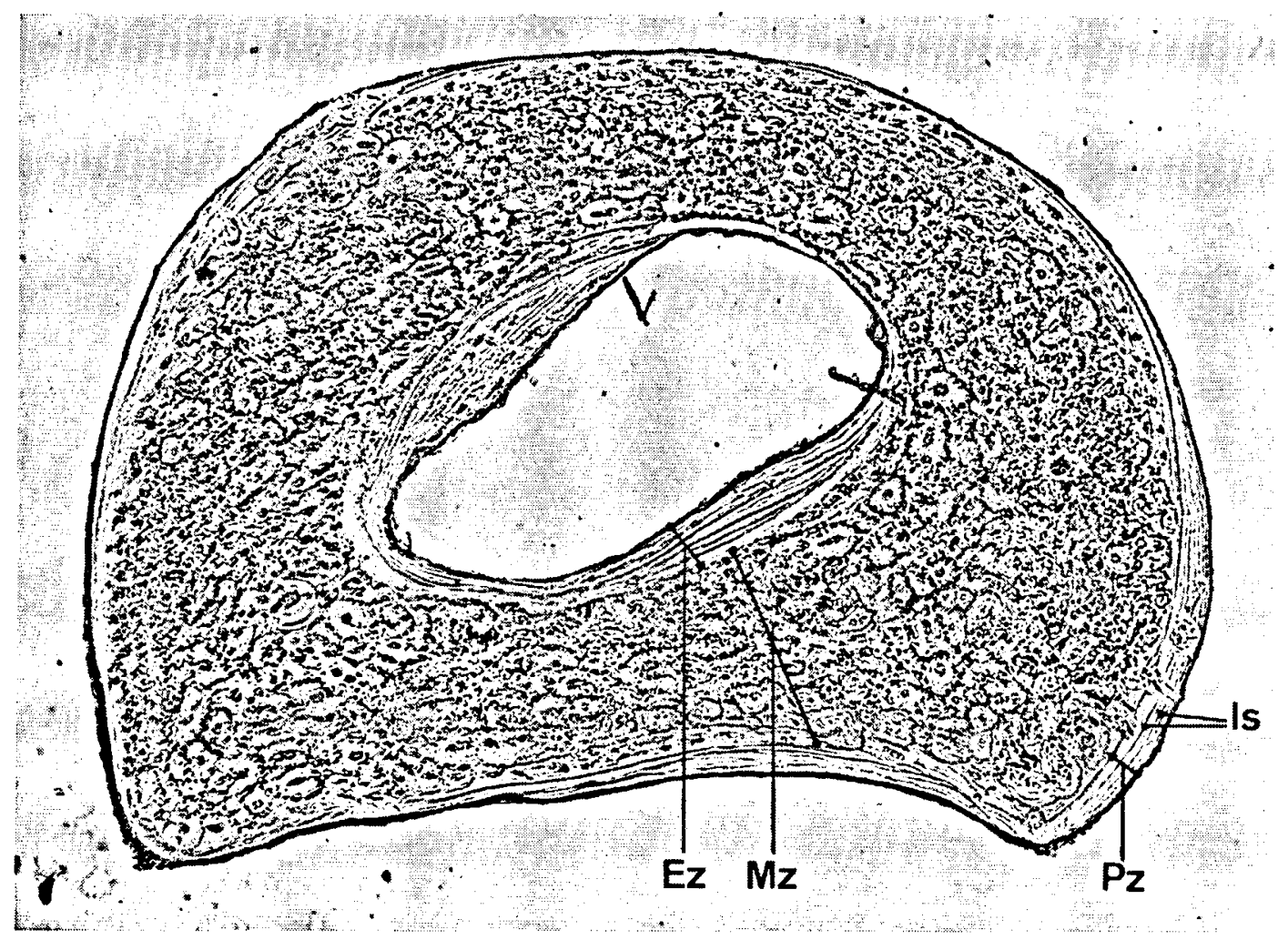

Fig. 1. Anas platyrhynchos, Duck, transverse ground down section of tibia. $\mathrm{PZ}=$ Periosteal zone, $\mathrm{MZ}=\mathrm{Mes}$ (asteal zone, $\mathrm{EZ}=$ Endosteal zone, $1 \mathrm{~s}=$ Layered structures.

by the farmer from which they were bought to be more than one season old. There was no information concerning the Jays, the Moorhen and the Duck.

The authors would like to express their gratitude towards Mr. C. Swennen of the Netherlands Institute of Sea Research (N.I.O.Z.) at Texel and to Drs J. Wattel, Curator of the department of Birds of the Zoological Museum of Amsterdam for supplying most of the material. They are very grateful to Miss C. M. King of the Animal Ecology Research Group of the University of Oxford for checking the manuscript, and to Mr. S. van Mechelen who produced the photographs.

As birds do not have teeth and while claws or bill are known to wear down rapidly, the only field of investigation left is the skeleton. Different parts of the skeleton are investigated by two methods, described by various mammalogists (see Klevezal \& Kleinenberg, 1969). The first method is to grind the bone down transversely on a preferably but not necessarily electrically operated - grinding disc, till a thin transparent section remains. The section is polished on carborundum until the required transparency is obtained and scratches in the surface have disappeared. Then the section is dehydrated and mounted in caedax on a microscopic slide. The results can be studied under a stereobinocular, a normal microscope or in polarized light. The second method consists of cutting thin sections of the bone, after it has been decalcified in $5 \% \mathrm{HNO}_{3}$ for $10-15$ hours and is impregnated with paraffin. The cut sections are then stained with a solution of haematoxylin of the following composition: $200 \mathrm{mg}$ haematoxylin, $2000 \mathrm{mg}$ Potassium-Aluminiumphosphate, $5 \mathrm{ml}$ ethanol $96 \%, 40 \mathrm{mg}$ sodiumiodate in $100 \mathrm{ml}$ distilled water; light haematoxylins are advisable, because slow staining produces best results and gives the opportunity to vary the duration of staining. The results can be studied under a normal microscope. Dry freezing is an easier and quicker method, and it can be used instead of paraffin impregnation. 
Table 1: Results of the age determination of several species of birds on the basis of counting the annual layers in the periosteal zone of bone.

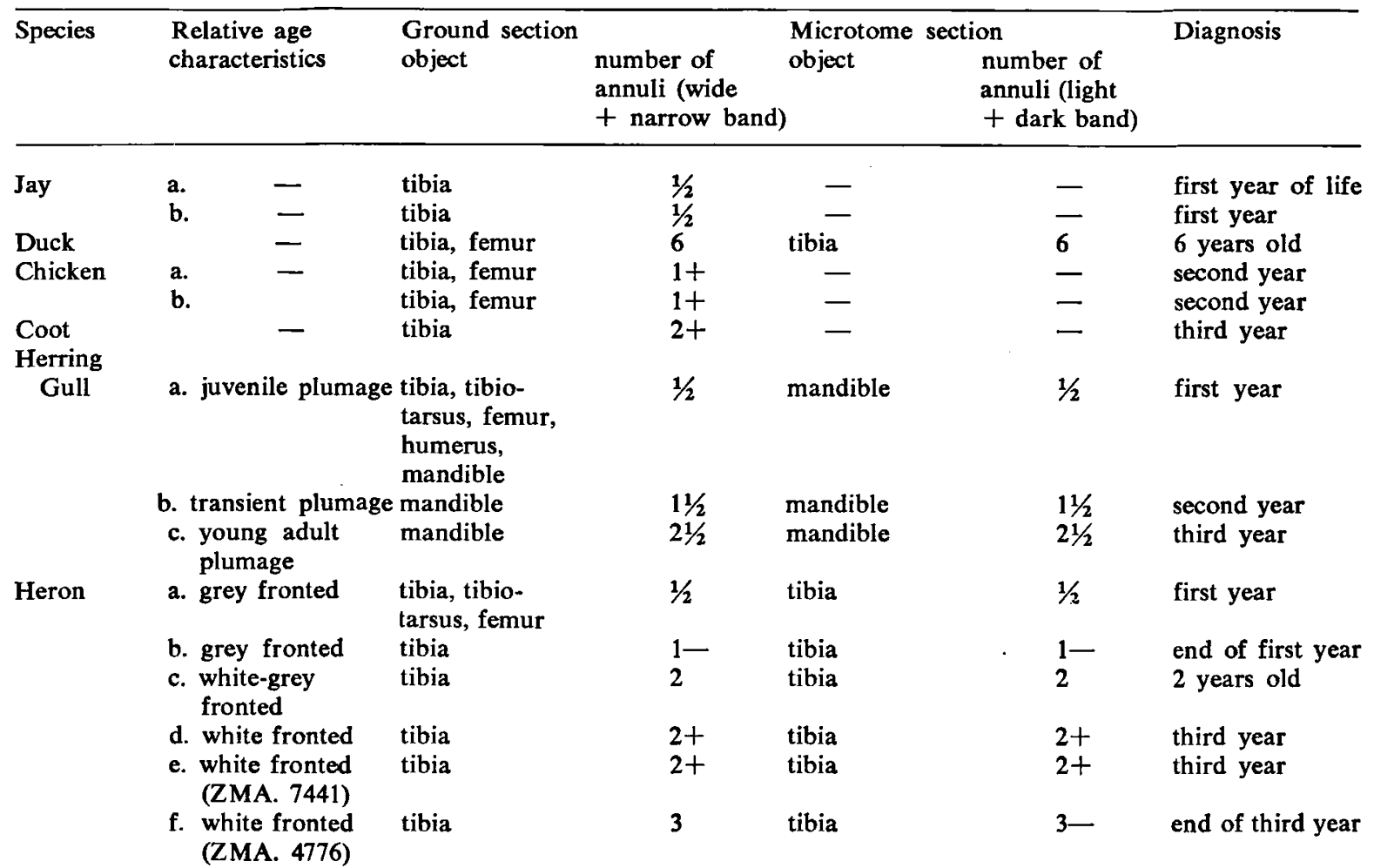

\section{RESULTS}

The results are summarized in table I and some of them are illustrated in the figures. The greatest difficulty proved to be in finding the most suitable locations in the skeleton. Not all parts of the skeleton show the same thickness of the periosteal zone. Best areas for examination are those on which tendons are inserted; apparently secondary deposition of bone is most intense in these places. With the majority of the investigated birds the distal part of the shaft of the tibia just over the epiphysis appears to be the most suitable place (Duck, Chicken, Moorhen, Heron); only with Herring Gulls this did not work out and here the most suitable part of bone is the mandible. The present investigations include most parts of the skeleton, e.g. femur, tibia, tibiotarsus, dactyli, crista, humerus, radius, mandible, clavicle, etc. The impression is formed that those parts which have to endure much mechanical load or tension are the parts in which the layers are well pronounced.

From the various figures given it can be seen that the periosteal and endosteal zones on the outer and inner side of the mesosteal zone are clearly distinguishable in most cases. Layered structures appear to be present in nearly all preparations. In those birds of which the age can be guessed from characteristics of their plumage a direct relation is found between age and number of narrow, c.q. wide zones in the periosteal zone. The Herring Gulls in the first year of their life do not possess a dark (narrow) layer, the animal in the second year of its life possesses one wide, one narrow and another wide layer, while the animal in the third year of its life possesses one wide, one narrow, another wide, another narrow and still another wide band. When it is assumed that a wide and a narrow zone represent the material deposited in one year the relation with the age is clear.

In the same way the Herons in their first year of life have only a wide zone (one specimen already has a narrow zone too), the Heron in its second year has a wide, a narrow and another complete wide band, while a second narrow zone is just being formed. The animals supposed to be over two years old all possess two wide and two narrow bands at least. 

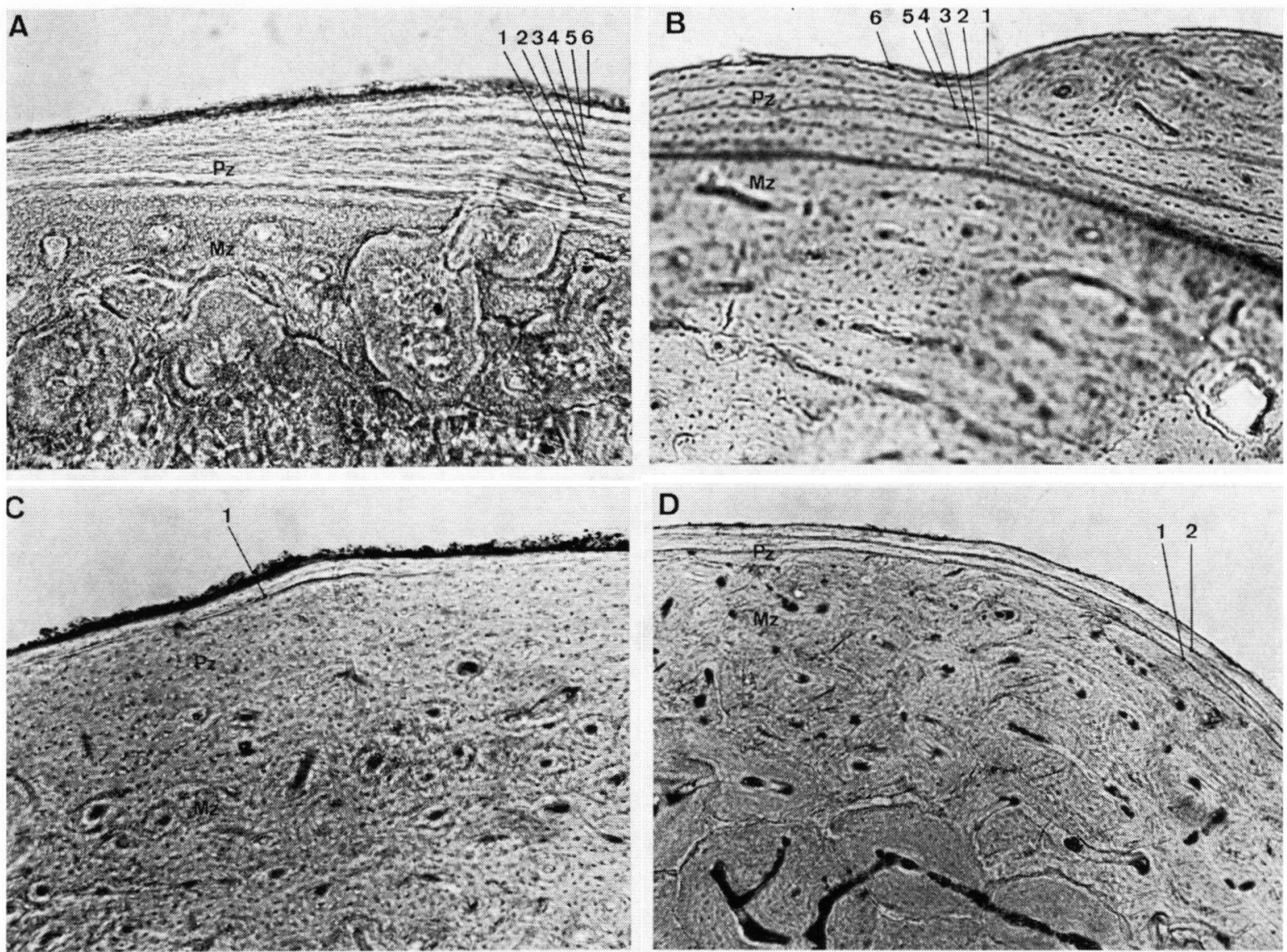

E

$\mathrm{F}$
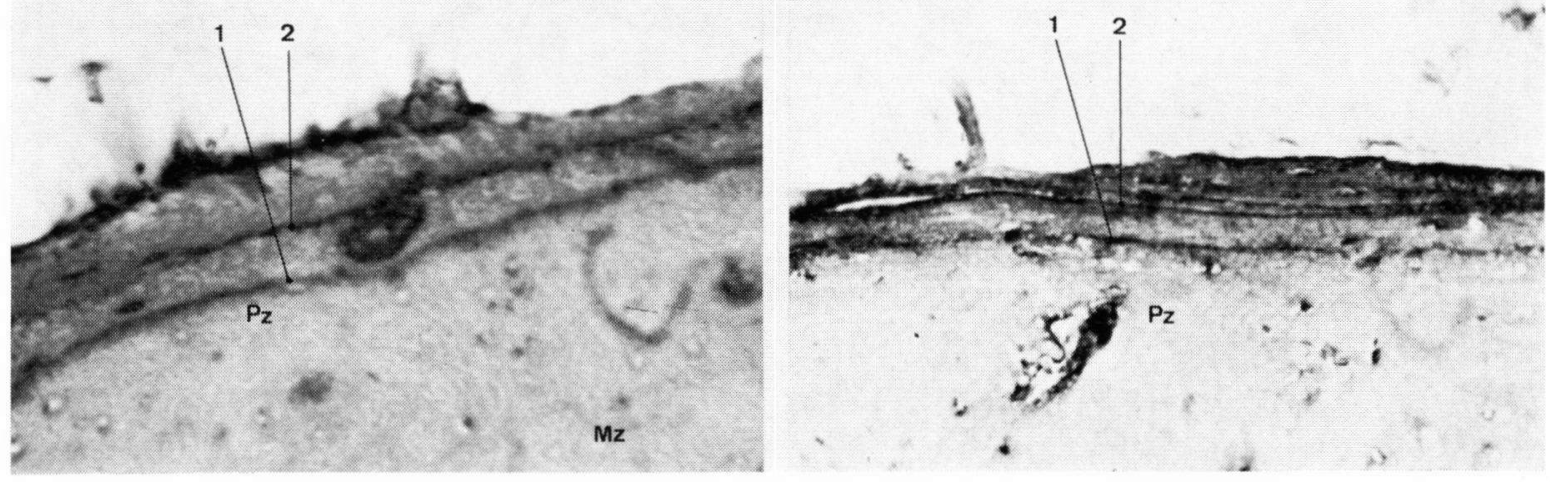

Fig. 2. A, Anas platyrhynchos, Duck, transverse ground down section of tibia (detail of fig. 1). Figures indicate supposed annual deposits (x 10).

B, Anas platyrhynchos, Duck, transverse microtome section of tibia, stained with haematoxylin solution (x 10).

C, Gallus gallus, Chicken, transverse ground down section of tibia (x 10).

D, Gallinula chloropus, Moorhen, transverse ground down section of tibia (x 10).

E, Larus argentatus, Herring Gull, third year specimen, transverse microtome section, stained with haematoxylin solution ( $x$ 40).

F, Ardea cinerea, Heron, specimen $d$, transverse microtome section of tibia, stained with haematoxylin solution (x 10). 
However, counting of annual layers might be somewhat more complicated. It often appears that the wide zone displays intermediary narrow zones, which are less clear and which represent presumably checks or decelerations during the period of fast growth. This is also the case with, for instance, dentinal growth layers in teeth of mammals. Various ecological factors may be responsible for this complication, which sometimes makes counting of annual layers difficult, particularly in older animals.

\section{DISCUSSION}

As was stated in the introduction it is, as yet, not possible to indicate the precise factors which are responsible for the deceleration and acceleration of the deposition of material in hard structures. Most authors consider environmental factors, and particularly seasonal climatic changes (wintersummer), as the most likely to cause the phenomenon described here (Klevezal \& Kleinenberg, 1969). In many cases it seems the best explanation. However, in Whales it has been demonstrated that apart from seasonal migration and periods of feeding alternating with fasting, also the ovulation has a definite influence on the growth of hard structures in females (e.g. Van Utrecht-Cock, 1966; Van Utrecht \& Van Utrecht-Cock, 1969). Recordings of baleen plates as well as photometric recordings of the layers in ear plugs show a relation of ovulation with certain growth accelerations. Male and female Fin Whales appear to have a different growth rhythm (Van Utrecht-Cock, 1966). Also sexual dimorphism in the deposition of the first dentinal layer ("neonatal" or "primary" line) in teeth has been discovered in Porpoises, Phocoena phocoena (L.) (Van Utrecht, 1969) and in Beech Martens, Martes foina (Erxleben) (Van Bree, Van Mensch \& Van Utrecht, 1971). Thus the influence of the sexual cycle on the growth of hard structures of any animal might be more substantial than is generally assumed.

As age determination is necessary to study the structure of a population, the method described in this paper might be an important contribution to avian ecology, because it is thought to be an absolute age indication, an advantage it can only share with bird banding. If age determination is only a minor object in a bird banding project, then this method should not be applied. However, if age determination is the major object of a bird banding project the method presented here may be considered as an alternative. Although a sample of the studied population must be killed in order to determine age in this way (which is not the case in bird banding), the results are obtained rapidly and with far less expenditure. If everything is worked out properly one should be able to obtain a reliable age pyramid of a population in one season.

When planning to study the age structure of a sample of a population using the method described here, one should select one older specimen to investigate the precise location in which the annual layers are seen best. The fact that in the species studied in this paper mostly the distal part of the tibia proved to be best, does not necessarily mean that this is also the case with other species.

In birds it could not be established with certainty whether there is an age at which no further layers of bone are deposited. In Fin Whales growth of baleen plates is continuous, but it is compensated by wear at the tip after a certain age. The same is true for the ear plugs in these animals to which the addition of material is also continuous, but after a certain age the layers at the tip are compressed together and form a very compact mass in which no layers are visible (Van Utrecht \& Van Utrecht-Cock, 1969). Up till now it cannot be demonstrated that periosteal growth in birds is not likewise submitted to compensatory wear or compression of layers. From the results obtained with the Duck (figs 1,2A and 2B) it can be concluded that animals of 6-7 years old still can be aged.

It will be observed from fig. 1 that also in the endosteal zone layers are visible. However, it is found that these are not always correlated in the same direct way with age as those found in the periosteal zone, probably because much calcium is resorbed from the endosteal zone during life (Klevezal \& Kleinenberg, 1969).

A possible application of this method may be in the study of age of fossil birds, considering the results that Peabody (1961) obtained with fossil fishes, amphibians and reptiles in this respect. 


\section{BIBLIOGRAPHY}

Bree, P. J. H. van, P. J. A. van Mensch \& W. L. van UTRECHT, 1971. Sur le dimorphisme sexuel des canines chez la Fouine, Martes foina (Erxleben, 1777). Mammalia, 34 (4) : ....

Klevezal, G. A. \& S. E. Kleinenberg, 1969. Age determination of mammals by layered structure in teeth and bone. (Akad. Nauk S.S.S.R., Inst. Morf. Zhiv., Izdatelstvo „Nauk”, Moscow: 1-144). Transl. Bur. for. Lang. Div. Dep. Secr. State Canada, Distr. Fish. Res. Board Canada, Transl. Ser., 1024 : 1-142.

LAws, R. M., 1952. A new method of age determination for mammals. Nature, 169 : 972.

Peabody, F. E., 1961. Annual growth zones in Living and Fossil Vertebrates. J. Morph., 108 : 11-62.
Petter-Rousseaux, A., 1953. Recherches sur la croissance et le cycle d'activité testiculaire de Natrix natrix helvetica (Lacépède). Terre et Vie, 100 (4) : 175-223.

UTRECHT, W. L. VAN, 1969. A remarkable feature in the dentine of teeth of Odontocetes. Beaufortia, 16 (217) : $157-162$.

UTREcht, W. L. Van \& C. N. Van Utrecht-CoCK, 1969. Comparison of records of baleen plates and of ear plugs in female Fin Whales, Balaenoptera physalus (Linnaeus, 1758). Bijdr. Dierk., $39: 81-97$.

UTrecht-Cock, C. N. van, 1966. Age determination and reproduction of female fin whales, Balaenoptera physalus (Linnaeus, 1758), with special regard to baleen plates and ovaries. Bijdr. Dierk., 35 : 39-100 (1965). 